\title{
Analysis of the classification of type-2 area in the zoning of environmental noise function
}

\author{
Su K.*, Liao M., Li L., Deng S., He Y. \\ Faculty of Geosciences and Environmental Engineering, Southwest Jiaotong University, Chengdu 611756, P. R. China \\ Received: 23/07/2021, Accepted: 13/09/2021, Available online: 21/09/2021
}

*to whom all correspondence should be addressed: e-mail: ksu@swjtu.edu.cn https://doi.org/10.30955/gnj.003877

\section{Graphical abstract}

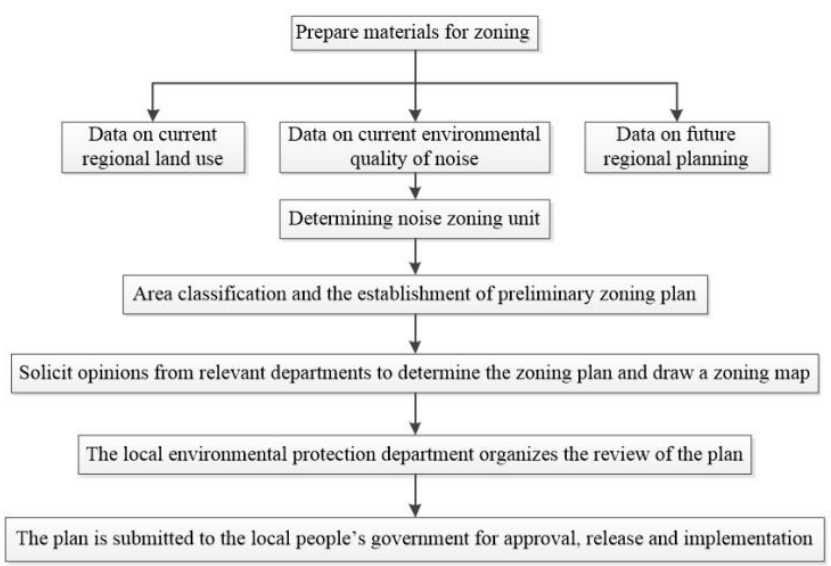

\begin{abstract}
The zoning of environmental noise function is aimed to provide a good acoustic environment for people. With the rapid urbanization, the zoning of environmental noise function has been widely conducted for China, and many first-tier and second-tier cities have made multiple zoning adjustments. Meanwhile, some third-and fourth-tier cities have also gradually implemented the zoning of environmental noise function. The method for determining the type-2 function area in the "Technical Specifications for Regionalizing Environmental Noise Function" (GB/T 151902014) is inapposite. In this work, the classification of the type- 2 area in different situations like the was discussed about the near boundary of the type- 0 area, type- 1 area, type-3area, and between type-1 area and type-3area. This work is expected to provide a certain reference for the zoning and adjustment of the environmental noise function areas in various places.
\end{abstract}

Keywords: Area of environmental noise function, zoning, type-2 function area of environmental noise, environmental noise management.

\section{Introduction}

With the social development and the progress of civilization, noise pollution has become a hot issue worldwide. The impact of noise on human health is well documented (Chen, 2020; Moudon, 2009). For China, in order to better implement the scientific outlook on development and build an ecological civilization, it is necessary to step up the management and protection of the urban acoustic environment (Xu, 2020). The zoning of environmental noise function can provide basis for environmental noise management, and facilitate relevant departments to propose targeted measures, thereby effectively controlling the degree and scope of noise pollution (Piccolo, 2005). This is of great significance for protecting and enhancing the environmental quality of noise (Burak, 2011; Nabil, 2019).

Nowadays, regionalizing environmental noise function involves mainly two aspects. One is to make the first zoning of environmental noise function in areas where the regionalization of environmental noise function has never been conducted; the other is to adjust the zoning of environmental noise function in areas where the regionalization of environmental noise function has been carried out many times. Whether it is the first regionalization or the adjustment of zoning, the Technical Specification for Regionalizing Environmental Noise Function (GB/T 15190-2014) is the core guiding document, which stipulates the principles and method of zoning.

In the Technical Specification for Regionalizing Environmental Noise Function (GB/T 15190-2014), type-2 area is defined as follows: type-0, type-1, and type- 3 function areas of environmental noise are determined first, and the remaining areas are classified into type-2 function areas of environmental noise. As is known to all, the higher level of the function area, the more efforts and money required to be invested in the management and protection of the acoustic environment. The method for the classification of the type-2 area mentioned in the specification is too general. In fact, it may include the area that should not be determined as the type-2 function area. This kind of zoning method causes a great loss of manpower and financial resources. Therefore, this paper discussed the determination of the type- 2 area in different situations, so as to provide a certain reference for the zoning and adjustment of environmental noise function in various places. 


\section{Methodology}

2.1. Main bases for regionalizing environmental noise function

"Law of the People's Republic of China on the Prevention and Control of Pollution from Environmental Noise", "Technical Specifications for Regionalizing Environmental Noise Function" (GB/T 15190-2014), "Environmental Quality Standard for Noise" (GB 3096), and "Classification of Urban Land Use and Planning Standards of Development Land" (GB50137-2011).

\subsection{Other bases}

The current status of urban and regional land use and urban planning, and the characteristics of regional pollution from environmental noise, and the requirements for urban environmental noise management.

\subsection{Steps for regionalizing environmental noise function}

At present, regionalizing environmental noise function is mainly based on the "Technical Specification for Regionalizing Environmental Noise Function" (GB/T 151902014), and the main steps are shown in Figure 1:

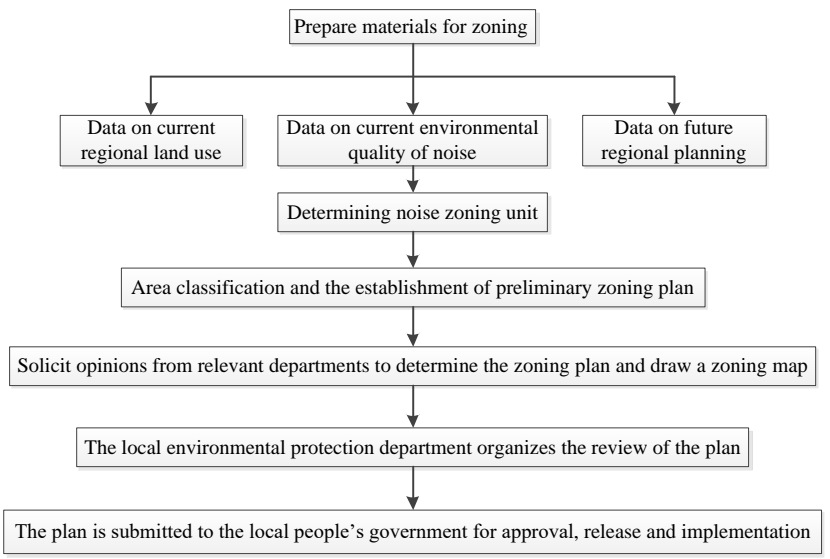

Figure 1. Steps for regionalizing environmental noise function.

During zoning and classification, it is required to connect multiple adjacent units with the same type of area to form a patch, and make full use of traffic arteries (main roads and above the level), district administrative boundaries, rivers, ravines, green spaces and other topographic features as zoning boundaries. With the scientific and technological progress, 3S (remote sensing technology, geographic information system, global positioning system technology), and ArcGIS software have also been applied to the zoning of environmental noise function. In Xiamen, the 3S technology was used to establish a spatial database for the zoning of environmental noise function, which achieved computer-based management of the regionalization of environmental noise function, and provided decision-making basis for the environmental noise management in Xiamen (Lin, 2011). Nanning (Li et al., 2012) and Shanyin County (Han et al., 2013) both applied ArcGIS software to their zoning project and achieved good outcomes. In the zoning of environmental noise function, the combination of theories and modern technologies can often improve the efficiency of zoning.

\section{Discussion}

3.1. Problems in the determination of the type-2 area in the zoning of environmental noise function

Type-2 function area of environmental noise refers to the area with commerce, finance and trade as the main functions, or where residence, commerce, and industry are mixed, and the residences need quietness. This area has higher requirements for noise control. According to the "Environmental Quality Standard for Noise" (GB 3096), the day and night noise limit in this area is $60 \mathrm{~dB}$ and $50 \mathrm{~dB}$, respectively. In the "Specification for Regionalizing Environmental Noise Function", the type-2 area is directly and generally defined as the area outside the type-0, type1 , and type- 3 function areas of environmental noise. Such zoning method is too general. A slight mistake in the classification of the type- 0 , type- 1 , and type- 3 areas will directly lead to the mistake in the determination of the type- 2 area. In addition, if there are some areas in the designated type-2 areas that can be classified into lowerlevel function areas of environmental noise such as type-3 areas, human and financial resources may be wasted in the later management. Therefore, considering a variety of factors, it found that the method for the classification of the type- 2 area in the Specification was inappropriate, thus this work explored how to determine the type-2 area in different locations.

As shown in Figure 2, the locations of the type-2 function area of environmental noise to be discussed are divided into two categories. One is located around a single classified area, and the other is located in the middle of two classified areas. In this paper, the area to be classified was discussed in different cases according to the type of the classified area.

(a)

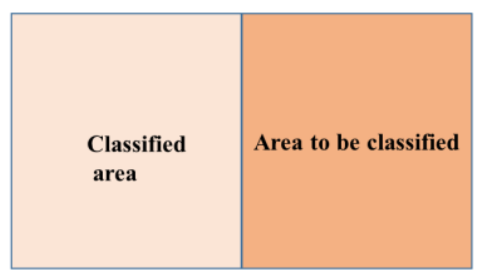

(b)

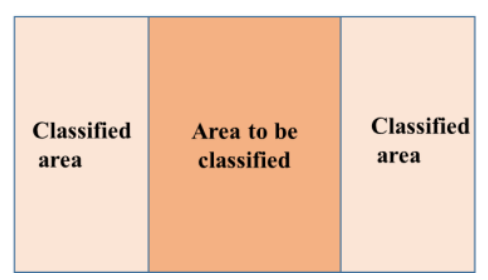

Figure 2. The location distribution map of the type-2 function area of environmental noise to be discussed. (a) Near a single classified area; (b) Between two classified areas.

\subsection{The classification of the type-2 area near the boundary} of the type-0 area

The type-0 function area of environmental noise is the area that requires quietness, such as rehabilitation area. Since there should be no obvious noise sources in and near the 
area, the boundary of the area is clear, and it is clearly stipulated that the type- 0 function area of environmental noise should avoid being close to the type- 3 and type- 4 areas in the Specification. Therefore, the unplanned area around this area should be determined as type- 2 area.

\subsection{The classification of the type-2 area near the boundary of the type-1 area}

Type- 1 area refers to the area where residence, health care, culture and education, scientific research and design, and administrative offices are the main functions and quietness is required. When the area to be classified is located next to a single type- 1 area and is not adjacent to any other classified type- 0 and type- 3 areas, considering the functions of the type- 1 area, the area to be divided should be classified into type- 2 area at this time, and improving the level of the function area can reduce the noise impact of this area on the adjacent type- 1 area. When the area to be classified is located between two or more type- 1 areas and is not adjacent to any other classified type- 0 and type- 3 areas, the area should also be classified into type-2 area.

\subsection{The classification of the type-2 area near the boundary of the type-3 area}

Type-3 function area of environmental noise refers to the area where industrial production, warehousing and logistics are the main functions, and the huge impact of industrial noise on the surrounding environment should be prevented.

When the area to be classified is located next to a single type- 3 area and is not adjacent to any other classified type0 and type- 1 areas, compared to the type- 1 area where the noise impact of the surrounding area needs to be controlled, the type-3 area produces certain noise pollution to the surrounding environment, therefore, if the surrounding area of the type- 3 area is directly classified into type- 2 area, the noise pollution of the type- 3 area compared to that of the type- 2 area needs to be considered. At this time, it is necessary to set up noise monitoring sites around the boundaries between the type3 area and the area to be classified and conduct corresponding detection. If the environmental quality of the noise in the surrounding type-2 area meets the standard, this zoning can be adopted, otherwise, control measures should be considered. Before adopting control measures, the number of people affected by the noise produced by these three types of areas should also be evaluated. If there are a large number of people affected, noise barriers can be built at the boundaries of the type- 3 area. If the number of people affected is small, noise insulation windows can be installed in a few sensitive buildings, which can better solve the noise pollution and save costs. In addition, if the area is a no-man's land, the scope of this area can be measured. If the local government has no planning for this area in the short term, or the planning is difficult to achieve in the short term, the area can be temporarily classified into type-3 area.

When the area to be classified is located between two or more type- 3 areas and is not adjacent to any other classified type- 0 and type- 1 areas, it should also be assessed whether there are populations or activities in this type- 2 area. If no obvious population activity or just a few population activities are found in the area, it is suggested to directly classify this area into type- 3 area. If the area to be divided is classified into type- 2 area due to the residence or activities of some people, first, as type- 3 area is represented by the industrial zone, corresponding noise reduction measures will be taken in this area, such as the development of new noise reduction technology, and noise-reduction facilities with noise barriers may be installed around the factories. Besides, the government will also invest considerable manpower for noise monitoring in this area. At this time, it is not worth paying high economic costs and a lot of manpower just to maintain the standard level of the environment quality of noise in a basically uninhabited area. Especially, when there are multiple areas to be classified near the type- 3 area, the economic cost may be doubled. For areas where just a small number of people live, noise insulation windows can be used to reduce noise and appropriate noise subsidies can be given to residents. This can basically solve the problem of noise pollution, and greatly save costs and achieve economical reduction of noise simultaneously.

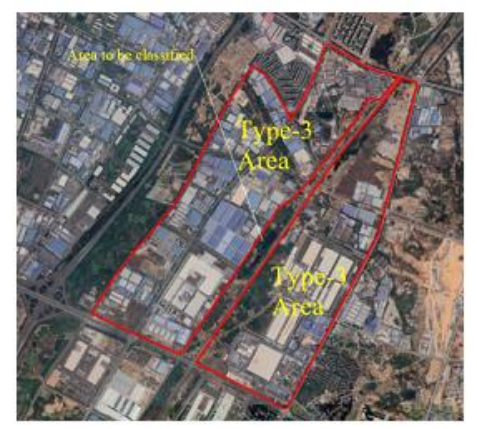

(a)

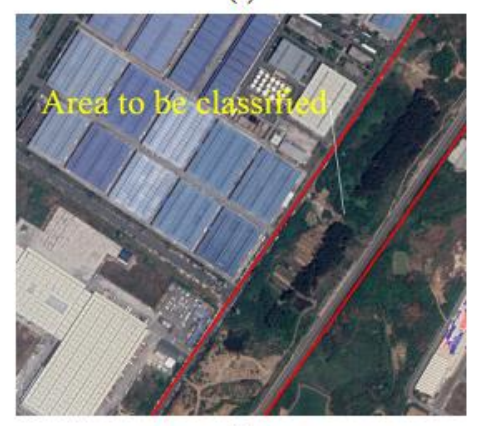

(b)

Figure 3. The case in Shuangliu District.(a) The relationship between the position of the area to be classified and the type-3 area; (b) A partial enlarged diagram of the area to be classified.

Figure 3 shows the classified type- 3 areas during the initial zoning of the environmental noise function in Shuangliu District of Chengdu. It can be seen that there is a long and narrow area to be classified between two type- 3 areas, and according to the Specification, this area should be defined as a type-2 area. However, through satellite images and actual survey, it was found that this area was uninhabited and few human activities were found, and there is no planning for this narrow and long area in the Shuangliu district. Therefore, Shuangliu professionals classified this 
area as a type- 3 area in the later adjustment. This example demonstrated that the determination of the type- 2 area should be based on specific conditions.

\subsection{The classification of the type-2 area between the type-} 1 and type- 3 areas

The classification of the area between the type- 1 area and the type- 3 area can be explored based on section 2.2 and 2.3 of this paper. First, the area between these two areas can be divided into two parts according to the ratio of the area of type- 1 to the area of type- 3 . The part close to the type- 1 area is determined as a type- 2 area; for the part near the type- 3 area, it supposes that the adjacent zone is first determined as the type- 2 area, and on this basis the noise impact of the type- 3 area compared to the adjacent type- 2 area is evaluated. If the evaluation result meets the standard, this part can be directly defined as a type- 2 area. Otherwise, it should be considered whether this area affects human living and activities. If it has such an influence, the area should be classified into type- 2 area and noise reduction measures should be conducted; if it has no such impact, and there is no planning for the area or the planning is difficult to realize in the short term, then this area is determined as a type- 3 area.

\section{Conclusion}

The area to be classified around the type- 0 and type- 1 areas of environmental noise function should be determined as type-2 area. In the classification of the adjacent area of the type- 3 area, it is necessary to consider the effect on the sensitive points near boundary between two areas. If evaluation result meets the standard, the objective area can be determined as a type- 2 area, or the impact on the living and activities of people should be evaluated. If it exerts certain influence, the area should be classified as a type- 2 area, and noise reduction measures need to be conducted; otherwise, there is no planning for this area or the planning is difficult to achieve in short term, and the area is defined as a type- 3 area; the cost of noise reduction should be considered in the determination of multiple type- 2 areas which are adjacent to type 3 areas. The areas with few people living can be classified as type-3 areas first, and then measures like noise insulation windows can be adopted for corresponding noise sensitive points. The area between type- 1 and type- 3 areas can be divided into two parts according to the ratio of the area of type- 1 to type-3. The part close to the type- 1 area is classified as a type- 2 area, and the part close to the type- 3 area is classified based on the principle of dividing the surrounding area of the type- 3 area. Additionally, the specificationd should be flexible as well as the measures in the classification of some special areas.

\section{Acknowledgement}

The authors acknowledge those who participated in the research, writing, revise, and reviews.

\section{References}

Burak U. (2011). Automatic environmental noise source classification model using fuzzy logic. Expert Systems with Applications, 38(7), 8751-8755.
Chengfeng L., Ying Q. and Yongying W. (2012). Regional environmental functional zoning in nanning city based on the ArcGIS. Inner Mongolia Environmental Sciences, 24(3), 70$71+40$. (In Chinese).

Guoli X. (2020). Study on the zoning of environmental noise function and management measures in Zhalantun City. Environment \& Development, 32(4), 206-208+210. (In Chinese).

Moudon A.V. (2009). Real noise from the urban environment: how ambient community noise affects health and what can be done about it. American Journal of Preventive Medicine, 37(2), 171.

Nabil M. and Sara M. (2019). Assessment of street-level noise in three different urban settings in Tripoli. Urban Climate, 29, 100481.

Piccolo A., Plutino D. and Cannistraro G. (2005). Evaluation and analysis of the environmental noise of Messina, Italy. Applied Acoustics, 66(4), 2005, 447-465.

Qingsong C. (2020). Pay attention to the prevention and control of noise and hand-transmitted vibration, and protect the health of workers. Journal of Environmental\&Occupational Medicine, 37(4), 334-336. (In Chinese).

Xu H., Bo L. and Jinyong H. (2013). Study on the environmental noise function zoning based on ArcGIS in Shanyin. Energy and Energy Conservation, (4), 79-80+83. (In Chinese).

Yunping L. (2011). Zoning of environmental noise function in Xiamen city based on 3s technology channel science, (6), 108109. (In Chinese). 